\title{
Effect of dietary vitamin $E$ on the performance of broilers and quality of broiler meat during refrigerated and frozen storage.
}

\author{
G.J.M. Coetzee and L.C. Hoffman ${ }^{\#}$ \\ Department of Animal Sciences, University of Stellenbosch, P. Bag X1, Matieland 7602, South Africa
}

\begin{abstract}
Experiment 1 was carried out with 220 one-day-old broiler chicks to evaluate the effect of 11 different levels of vitamin $\mathrm{E}$ supplementation (viz. 0 to $200 \mathrm{mg} \alpha$-tocopheryl acetate $/ \mathrm{kg}$ diet) on the production performance of broilers and oxidative stability of frozen broiler carcasses. The diets with vitamin E levels of 0 to $100 \mathrm{mg}$ were fed from day-old to 42 days of age, and the diets with vitamin E levels of 120 to $200 \mathrm{mg}$ were fed from 21 to 42 days of age. The oxidative stability, evaluated by thiobarbituric acid reactive substances (TBARS), was determined after $30,90,120$ and 150 days of storage at $-20{ }^{\circ} \mathrm{C}$. There were no differences in weight gain $(2.29 \pm 0.397 \mathrm{~kg})$ or feed conversion ratios $(1.85 \pm 0.111 \mathrm{~kg}$ feed $/ \mathrm{kg}$ gain $)$ between dietary treatments. TBARS values increased with increasing time of storage (basal diet: day $30=1.71 \pm$ 0.51 ; day $150=4.89 \pm 0.51$ ), but decreased with increasing vitamin $E$ levels (day 150 : basal $=4.89 \pm 0.51$; $100 \mathrm{mg} / \mathrm{kg}=1.09 \pm 0.27$ ). Experiment 2 was carried out with day-old broiler chicks to evaluate the effect of five levels of vitamin E supplementation (viz. 0 to $160 \mathrm{mg} \alpha$-tocopheryl acetate $/ \mathrm{kg}$ diet) on performance parameters and the oxidative stability of refrigerated carcasses. The experimental diets were fed from dayold to 42 days of age. Oxidative stability, evaluated by TBARS, colour deterioration and microbiological stability was determined after $0,4,8,10$ and 12 days of storage at $4{ }^{\circ} \mathrm{C}$. Fatty acid analysis was done on samples obtained on days 0 and 12 . There were no differences in total weight gain $(2.37 \pm 0.467 \mathrm{~kg})$ or feed conversion ratio $(1.88 \pm 0.117 \mathrm{~kg}$ feed $/ \mathrm{kg}$ gain) between dietary treatments. TBARS values increased with increasing storage time, but decreased with increasing vitamin $\mathrm{E}$ levels. There were no differences between treatments for colour measurements for $\mathrm{L}^{*}(44.97 \pm 0.662)$, $\mathrm{a}^{*}(5.23 \pm 0.315)$ or $\mathrm{b}^{*}(12.76 \pm 0.321)$ values. Microbiological counts increased over time, but dietary vitamin E concentration had no effect. There were no differences between dietary treatments for any of the groups of fatty acids measured (SFA: Day $0=26.1 \pm$ $1.13 \%$, Day $12=26.1 \pm 1.17 \%$; MUFA: Day $0=41.4 \pm 1.46 \%$, Day $12=40.2 \pm 2.28 \%$; PUFA: Day $0=$ $32.4 \pm 1.95 \%$, Day $12=33.8 \pm 2.52 \%$ ). Similarly, fatty acid proportions did not change over time. There were no differences between dietary groups for mean muscle $\mathrm{pH}(6.01 \pm 0.206)$.
\end{abstract}

Keywords: broiler meat, Vitamin E, TBA, fatty acids, shelf life, refrigeration, frozen storage, poultry

\# Author to whom correspondence should be addressed; e-mail: lch@maties.sun.ac.za

\section{Introduction}

Lipid oxidation is a major cause of meat quality deterioration, as products of autoxidation of unsaturated fatty acids affect wholesomeness and nutritional value (Pearson et al., 1983). Lipid oxidation is an important determinant of shelf life of meats and meat products. Post-slaughter biochemical changes involved in the conversion of muscle to meat are accompanied by a loss of cellular antioxidant defences and an increased propensity of meat lipids to undergo oxidation (Morrisey et al., 1994). This contributes to undesirable changes in a number of quality parameters, including loss of water-holding capacity, texture and flavour.

The lipids in poultry exhibit a higher degree of unsaturation than red meats due to a relatively high content of phospholipids (Igene \& Pearson, 1979). The degree of unsaturation of the phospholipids of the subcellular membrane is an important factor in determining the oxidative stability of meats, with the oxidative potential increasing as the degree of unsaturation of the lipids in the meat increases. The phospholipids are located in membrane structures, and the initiation of lipid peroxidation in tissues has been tracked to the membrane level (Machlin, 1984). The production of meat, particularly chicken, with a more highly unsaturated profile has been the focus of some attention, as such meats are perceived as having a 'healthier' image. Accordingly, poultry meat and meat products are rather susceptible to oxidative deterioration, and oxidation often determines the shelf life of poultry meat products.

Membrane lipids are protected against oxidative attack by a number of natural occurring antioxidants, including the chain-breaking antioxidants ascorbic acid and $\alpha$-tocopherol. $\alpha$-Tocopherol (vitamin E) is 
deposited in subcellular membranes in positions adjacent to the phospholipid chains and is a highly efficient scavenger of neighbouring fatty acyl radicals (Buettner, 1993). The addition of $\alpha$-tocopherol to animal diets is an effective means of improving the oxidative stability of meats, in addition to improving flavour (Sheldon et al., 1997). Elevated $\alpha$-tocopherol levels in poultry feeds increase tissue concentrations thereof resulting in improved stability of membrane structures which may be expected to increase the oxidative stability of meat and meat products (Sheehy et al., 1993).

Feeding poultry a higher level of natural dietary antioxidants provides the poultry industry with a simple method for improving oxidative stability, sensory quality, shelf life, and acceptability of poultry meats (Buckley \& Morrissey, 1992). In addition to the stabilising effect on meat and meat products, a raised $\alpha$-tocopherol level significantly enhances feed conversion efficiency, average weight, and net income per bird (Kennedy et al., 1992).

The purpose of the present study was to investigate the effect of vitamin E supplementation on quality attributes in chicken meat following refrigerated and frozen storage.

\section{Materials and Methods}

At the start of experiment 1, 220 one-day-old Ross broilers were reared to 42 days of age in an environmentally controlled rearing house. A 24-hour light period was followed. The initial environmental temperature at day-old was $33^{\circ} \mathrm{C}$, which was gradually decreased to $21^{\circ} \mathrm{C}$ at 21 days of age according to the Ross manual. A standard commercial health and inoculation program was followed. Five birds per cage were allocated to 44 cages. All the birds in each cage were allocated to one of eleven dietary treatments. In experiment 2,100 one-day-old broilers were used. All rearing procedures were the same as for experiment 1 . Five birds per cage were allocated to 20 cages. All the birds in each cage were allocated to one of five dietary treatments. A randomised block design was used in both experiments.

The nutrient composition of the experimental diets is given in Table 1. In experiment 1, ten levels of vitamin E (supplied by Hoffmann La-Roche, Basel, Switzerland) were supplemented to the basal diet (Table 2), resulting in 11 treatments: 1) basal diet (control); 2) basal $+20 \mathrm{mg}$ Vitamin E/kg feed; 3) basal $+40 \mathrm{mg}$ Vitamin E / $\mathrm{kg}$ feed; 4) basal $+60 \mathrm{mg}$ Vitamin E / $\mathrm{kg}$ feed; 5) basal + $80 \mathrm{mg}$ Vitamin E $/ \mathrm{kg}$ feed; 6) basal + $100 \mathrm{mg}$ Vitamin E / $\mathrm{kg}$ feed; 7) basal + $120 \mathrm{mg}$ Vitamin E / kg feed; 8) basal + $140 \mathrm{mg}$ Vitamin E $/ \mathrm{kg}$ feed; 9) basal + $160 \mathrm{mg}$ Vitamin E / kg feed; 10) basal + $180 \mathrm{mg}$ Vitamin $\mathrm{E} / \mathrm{kg}$ feed; 11) basal $+200 \mathrm{mg}$ Vitamin E $/ \mathrm{kg}$ feed. Treatments 2 to 6 were fed from day-old to 42 days of age (throughout the trial), while treatments 7 to 11 were fed from 21 to 42 days of age (last three weeks of the trial).

Table 1 Calculated nutrient composition of experimental diets

\begin{tabular}{lc}
\hline Nutrient & Percentage of diet \\
\hline Metabolisable Energy (MJ/kg) & 13.20 \\
Protein & 22.9 \\
Arginine & 1.45 \\
Isoleucine & 0.98 \\
Leucine & 2.38 \\
Lysine & 1.15 \\
Methionine & 0.57 \\
Methionine + Cystine & 0.95 \\
Threonine & 0.77 \\
Tryptophan & 0.23 \\
Calcium & 0.89 \\
Sodium & 0.16 \\
Phosphorus & 0.40 \\
Moisture & 9.20 \\
\hline
\end{tabular}

In experiment 2, four levels of vitamin $\mathrm{E}$ were supplemented to the basal diet (Table 2), resulting in five treatments: 1) basal diet (control); 2) basal $+40 \mathrm{mg}$ Vitamin $\mathrm{E} / \mathrm{kg}$ feed; 3) basal $+80 \mathrm{mg}$ Vitamin $\mathrm{E} / \mathrm{kg}$ feed; 4) basal + $120 \mathrm{mg}$ Vitamin E $/ \mathrm{kg}$ feed; 5) basal + $160 \mathrm{mg}$ Vitamin E $/ \mathrm{kg}$ feed. All treatments were fed from day-old to 42 days of age. Bird weights per cage and feed consumption per cage were recorded weekly. Weight gain (WG) and feed conversion ratios (FCR) were calculated. 
Table 2 The formulation of the basal diet

\begin{tabular}{lc}
\hline Ingredient & Percentage of diet \\
\hline Maize gluten 60 & 8.2 \\
Feed lime & 1.3 \\
Coccidiostat & 0.2 \\
Lysine & 0.5 \\
Maize & 51.2 \\
Mono-dicalciumphosphate & 1.3 \\
Synthetic methionine & 0.2 \\
Soybean meal & 17.6 \\
Fine salt & 0.4 \\
Sunflower meal & 10.9 \\
Vitamin/Mineral Mix ${ }^{1}$ & 0.4 \\
Oil mixture & 8.0 \\
\hline
\end{tabular}

${ }^{1}$ Vitamin/Mineral mix: Vitamin A (10 000000 IU), Vitamin D (2 000000 IU), Vitamin E (20 000 IU), Vitamin K (2 g), Vitamin B1 (2 g), Vitamin B2 (5 g), Vitamin B6 (3 g), Vitamin B12 (0.01 g), Niacin (20 g), Calpan DL (10 g), Folic acid (0.5 g), Biotin (0.02 g), Antioxidant (125 g), Choline (300 g), Cobalt (0.5 g), Copper (6 g), Iron (20 g), Manganese (70 g), Iodine ( $1 \mathrm{~g})$, Selenium (0.15 g), Zinc (50 g).

Five birds from each cage were slaughtered at 42 days of age. The weight of the plucked birds was recorded and the abdominal fat pads removed. The five carcasses from each cage were stored in separate oxygen permeable plastic bags at $-20{ }^{\circ} \mathrm{C}$ (experiment 1) or $4{ }^{\circ} \mathrm{C}$ (experiment 2) until required for chemical analysis. In experiment 1 (frozen storage) one carcass from each cage was taken from storage on days 30, 90, 120 and 150, minced and oxidative stability measured by means of TBARS values (Uchiyama \& Mihara, 1978). In experiment 2 (refrigerated storage) $\mathrm{pH}$ measurements were taken $45 \mathrm{~min}, 2,4$ and $24 \mathrm{~h}$ postmortem. One carcass from each cage was taken from storage on days $0,4,8,10$ and 12 , measured for colour degradation, minced and analysed for microbial spoilage and oxidative stability by means of TBARS values. Fresh meat colour of the muscle was evaluated using a Colorgard System 2000 colorimeter (Pacific Scientific, Silver Spring, MD, USA) to determine $\mathrm{L}^{*}, \mathrm{a}^{*}$ and $\mathrm{b}^{*}$ values (Commission International de 1' Eclairage, 1976), with $\mathrm{L}^{*}$ indicating brightness, $\mathrm{a}^{*}$ the red-green range and $\mathrm{b}^{*}$ the blue-yellow range. Fatty acid analysis was done on the samples obtained on days 0 and 12. Fatty acid methyl esters (FAME) were prepared according to the method of Morrison \& Smith (1964). The FAME were analysed by means of GLC on a Varian Model 3300, equipped with flame ionisation detection and two $30 \mathrm{~m}$ fused silica megabore DB225 columns of $0.53 \mathrm{~mm}$ internal diameter (J\&W Scientific, Folsom, CA). Gas flow rates were: hydrogen, $25 \mathrm{ml} / \mathrm{min}$; air, $250 \mathrm{ml} / \mathrm{min}$; and nitrogen (carrier gas), $5-8 \mathrm{ml} / \mathrm{min}$. Temperature programming was linear at $4^{\circ} \mathrm{C} / \mathrm{min}$; initial temperature, $160{ }^{\circ} \mathrm{C}$; final temperature, $220^{\circ} \mathrm{C}$ held for $10 \mathrm{~min}$; injector temperature, 240 ${ }^{\circ} \mathrm{C}$; and detector temperature, $250{ }^{\circ} \mathrm{C}$. The FAME were identified by comparison of the retention times to those of a standard FAME mixture (Nu-Chek-Prep Inc., Elysian, Minnesota). Microbial stability was measured on the samples of days $0,4,8$ and 10 by means of aerobic plate counts (APC). Total aerobic microbial counts were conducted by using Plate Count Agar (Merck). Ten grams of meat were homogenised in $100 \mathrm{ml}$ of sterile distilled water, serially diluted and plated onto the surface of the plates. All plates were incubated at $37{ }^{\circ} \mathrm{C}$ for 48 to $72 \mathrm{~h}$. In a separate experiment, $100 \mathrm{~g}$ fresh meat samples with vitamin E levels of 0,80 and $160 \mathrm{mg}$ vitamin $\mathrm{E} / \mathrm{kg}$ feed were inoculated with active growing cells of Listeria innocua, Staphylococcus aureus and Salmonella enteritidis $\left(10^{6} \mathrm{CFU} / \mathrm{g}\right)$. The growth of these bacteria was monitored by plating onto Trypticase Soy Agar (Merck), Baird-Parker Agar (Merck) and Salmonella-Shigella Agar (Merck), respectively. All plates were incubated at $37{ }^{\circ} \mathrm{C}$ for 48 to $72 \mathrm{~h}$.

The GENSTAT statistical package (GENSTAT 5 Release 3.2, 1997) was used to fit regression models to the data with storage time as predictor. Analysis of variance was performed on all variables measured. The following model was fitted for main effects (diet, storage time) and the interaction between them:

$$
Y_{i j}=\mu+D_{i}+T_{j}+D T_{i j}+e_{i j}
$$

where $Y_{i j}$ is the dependant variable, $\mu=$ the overall mean, $D_{i}=$ the $i^{\text {th }}$ diet effect, $T_{j}=$ the $j^{\text {th }}$ time effect, $D T_{i j}$ $=$ the interaction between diet and time and $\mathrm{e}_{\mathrm{ij}}=$ the residual error. The differences between diets and storage 
times were tested separately by means of the null hypothesis $\left(\mathrm{H}_{0}\right)$, with $\mathrm{H}_{0}: \mu=\mu_{0}$ and the alternate hypothesis $\left(\mathrm{H}_{\mathrm{a}}\right)$ being $\mathrm{H}_{\mathrm{a}}: \mu \neq \mu_{0}$. Mean and standard errors were calculated for each diet and time and the main effects were compared by use of least significant differences. Differences between the variables were accepted as being significant if the probability of rejection of $\mathrm{H}_{0}$ was less than $5 \%(\mathrm{P}<0.05)$ for diets and time.

\section{Results}

The weight gain (WG) and feed conversion ratio (FCR) of chicks fed the different experimental diets for experiments 1 and 2 are shown in Table 3. There were no differences $(P>0.05)$ in BWG or FCR between the dietary treatments for either of the experiments.

Table 3 Effects of various dietary vitamin E levels on weight gain $(\mathrm{kg})$ and feed conversion ratios of broilers at 42 days of age (mean \pm s.e.)

\begin{tabular}{lcc}
\hline Vitamin E concentrations & FCR & WG $(\mathrm{kg})$ \\
\hline Experiment $1\left(-20^{\circ} \mathrm{C}\right)$ & & \\
1) Basal & $1.79 \pm 0.173$ & $2.32 \pm 0.434$ \\
2) $20 \mathrm{mg}$ vitamin $\mathrm{E} / \mathrm{kg}$ feed & $1.84 \pm 0.137$ & $2.24 \pm 0.223$ \\
3) $40 \mathrm{mg}$ vitamin $\mathrm{E} / \mathrm{kg}$ feed & $1.91 \pm 0.067$ & $2.28 \pm 0.230$ \\
4) $60 \mathrm{mg}$ vitamin $\mathrm{E} / \mathrm{kg}$ feed & $1.90 \pm 0.024$ & $2.05 \pm 0.130$ \\
5) $80 \mathrm{mg}$ vitamin $\mathrm{E} / \mathrm{kg}$ feed & $1.83 \pm 0.056$ & $2.11 \pm 0.041$ \\
6) $100 \mathrm{mg}$ vitamin $\mathrm{E} / \mathrm{kg}$ feed & $1.82 \pm 0.063$ & $2.19 \pm 0.335$ \\
7) $120 \mathrm{mg}$ vitamin $\mathrm{E} / \mathrm{kg}$ feed & $1.84 \pm 0.107$ & $2.56 \pm 0.684$ \\
8) $140 \mathrm{mg}$ vitamin $\mathrm{E} / \mathrm{kg}$ feed & $1.81 \pm 0.083$ & $2.16 \pm 0.086$ \\
9) $160 \mathrm{mg}$ vitamin $\mathrm{E} / \mathrm{kg}$ feed & $1.94 \pm 0.197$ & $2.53 \pm 0.618$ \\
10) $180 \mathrm{mg}$ vitamin $\mathrm{E} / \mathrm{kg}$ feed & $1.75 \pm 0.107$ & $2.44 \pm 0.631$ \\
11) $200 \mathrm{mg}$ vitamin $\mathrm{E} / \mathrm{kg}$ feed & $1.87 \pm 0.163$ & $2.33 \pm 0.449$ \\
& & \\
Experiment $2\left(4{ }^{\circ} \mathrm{C}\right)$ & & \\
1) $\mathrm{Basal}$ & $1.90 \pm 0.072$ & $2.33 \pm 0.173$ \\
2) $40 \mathrm{mg}$ vitamin $\mathrm{E} / \mathrm{kg}$ feed & $1.89 \pm 0.073$ & $2.34 \pm 0.240$ \\
3) $80 \mathrm{mg}$ vitamin $\mathrm{E} / \mathrm{kg}$ feed & $1.85 \pm 0.062$ & $2.12 \pm 0.035$ \\
4) $120 \mathrm{mg}$ vitamin $\mathrm{E} / \mathrm{kg}$ feed & $1.84 \pm 0.131$ & $2.70 \pm 0.756$ \\
5) $160 \mathrm{mg}$ vitamin $\mathrm{E} / \mathrm{kg}$ feed & $1.98 \pm 0.223$ & $2.66 \pm 0.680$ \\
\hline
\end{tabular}

The TBARS values for experiment 1 and 2 are given in Table 4. TBARS values increased $(\mathrm{P}<0.05)$ for both experiments with increasing time of storage (Figures 1 and 2). Regression equations were fitted to the data and both experiments showed significant linear equations for oxidation over time (Table 5).

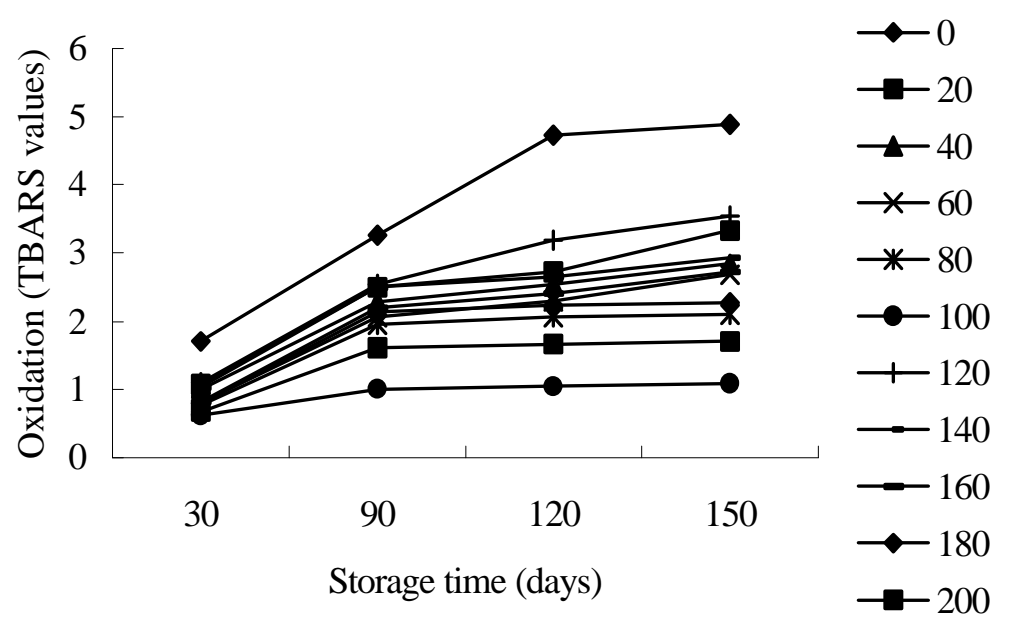

Figure 1 The effect of different levels of vitamin $\mathrm{E}$ (mg vitamin $\mathrm{E} / \mathrm{kg}$ feed) on the oxidative stability (TBARS) of broiler carcasses during storage at $-20{ }^{\circ} \mathrm{C}$ for up to five months 
Table 4 The effect of storage time on the oxidative stability (TBARS values $-\mathrm{mg}$ malondialdehyde/kg tissue) of broiler carcasses from birds fed different levels of vitamin $\mathrm{E}$ (mean \pm s.e.)

\begin{tabular}{|c|c|c|c|c|c|}
\hline $\begin{array}{l}\text { Vitamin E concentration } \\
\text { Experiment } 1\left(-20^{\circ} \mathrm{C}\right) \\
\text { Supplemented throughout }\end{array}$ & Day 30 & Day 90 & $\begin{array}{c}\text { TBA absorbance } \\
\text { Day } 120\end{array}$ & Day 150 & \\
\hline 1) Basal & $1.71_{a}{ }^{e} \pm 0.51$ & $3.27_{b}^{f} \pm 0.51$ & $4.73_{\mathrm{c}}{ }^{\mathrm{i}} \pm 0.51$ & $4.89_{c}^{f} \pm 0.51$ & \\
\hline 2) $20 \mathrm{mg}$ vitamin $\mathrm{E} / \mathrm{kg}$ feed & $1.07 \mathrm{ad} \pm 0.07$ & $2.50_{b}^{b} \pm 0.07$ & $2.72_{c}^{\mathrm{d}} \pm 0.07$ & $3.33_{\mathrm{d}}^{\mathrm{b}} \pm 0.07$ & \\
\hline 3) $40 \mathrm{mg}$ vitamin $\mathrm{E} / \mathrm{kg}$ feed & $1.00^{\mathrm{bcd}} \pm 0.10$ & $2.29_{b}^{b c} \pm 0.10$ & $2.54_{c}^{\mathrm{cdh}} \pm 0.10$ & $2.84_{d}^{c} \pm 0.10$ & \\
\hline 4) $60 \mathrm{mg}$ vitamin $\mathrm{E} / \mathrm{kg}$ feed & $0.81_{\mathrm{a}}^{\mathrm{ab}} \pm 0.10$ & $2.06_{b}{ }^{c d} \pm 0.10$ & $2.30_{\mathrm{c}}^{\mathrm{efh}} \pm 0.10$ & $2.68_{d}^{c} \pm 0.10$ & \\
\hline 5) $80 \mathrm{mg}$ vitamin $\mathrm{E} / \mathrm{kg}$ feed & $0.76_{\mathrm{a}}^{\mathrm{ac}} \pm 0.05$ & $1.96_{b}^{d} \pm 0.05$ & $2.06_{\mathrm{c}}^{\mathrm{f}} \pm 0.05$ & $2.10_{c}^{\mathrm{d}} \pm 0.05$ & \\
\hline 6) $100 \mathrm{mg}$ vitamin $\mathrm{E} / \mathrm{kg}$ feed & $0.62_{\mathrm{a}}^{\mathrm{a}} \pm 0.27$ & $1.00_{a b}^{d} \pm 0.27$ & $1.05_{\mathrm{b}}^{\mathrm{a}} \pm 0.27$ & $1.09_{b}^{a} \pm 0.27$ & \\
\hline Supplemented last 3 weeks & & & & & \\
\hline 7) $120 \mathrm{mg}$ vitamin $\mathrm{E} / \mathrm{kg}$ feed & $1.10_{\mathrm{a}}^{\mathrm{b}} \pm 0.03$ & $2.54_{b}^{b} \pm 0.03$ & $3.18_{c}^{b} \pm 0.03$ & $3.54_{d}^{b} \pm 0.03$ & \\
\hline 8) $140 \mathrm{mg}$ vitamin $\mathrm{E} / \mathrm{kg}$ feed & $1.03_{\mathrm{a}}^{\mathrm{bc}} \pm 0.04$ & $2.50_{\mathrm{b}}^{\mathrm{b}} \pm 0.04$ & $2.65_{\mathrm{c}}^{\mathrm{cd}} \pm 0.04$ & $2.94_{d}^{c} \pm 0.04$ & \\
\hline 9) $160 \mathrm{mg}$ vitamin $\mathrm{E} / \mathrm{kg}$ feed & $0.82^{a}{ }^{a b} \pm 0.15$ & $2.20_{\mathrm{b}}{ }^{\mathrm{cd}} \pm 0.15$ & $2.41_{b}{ }^{c e} \pm 0.15$ & $2.72_{c}^{c} \pm 0.15$ & \\
\hline 10) $180 \mathrm{mg}$ vitamin $\mathrm{E} / \mathrm{kg}$ feed & $0.79^{a} a^{\text {acd }} \pm 0.09$ & $2.14_{b}^{c d} \pm 0.09$ & $2.24_{b c}{ }^{\text {ef }} \pm 0.09$ & $2.28_{c}^{d} \pm 0.09$ & \\
\hline 11) $200 \mathrm{mg}$ vitamin $\mathrm{E} / \mathrm{kg}$ feed & $0.67_{\mathrm{a}}^{\mathrm{a}} \pm 0.26$ & $1.61_{\mathrm{b}}^{\mathrm{e}} \pm 0.26$ & $1.66_{\mathrm{b}}^{\mathrm{g}} \pm 0.26$ & $1.70_{\mathrm{b}}^{\mathrm{e}} \pm 0.26$ & \\
\hline Experiment $2\left(4^{\circ} \mathrm{C}\right)$ & Day 0 & Day 4 & Day 8 & Day 10 & Day 12 \\
\hline 1) Basal & $0.98_{a}^{a} \pm 0.12$ & $1.53_{\mathrm{b}}^{\mathrm{d}} \pm 0.23$ & $3.099_{c}^{d} \pm 0.23$ & $4.56_{\mathrm{d}}^{\mathrm{e}} \pm 0.24$ & $4.72_{\mathrm{d}}^{\mathrm{e}} \pm 0.24$ \\
\hline 2) $40 \mathrm{mg}$ vitamin $\mathrm{E} / \mathrm{kg}$ feed & $0.899^{a} \pm 0.08$ & $1.10_{\mathrm{b}}^{\mathrm{a}} \pm 0.02$ & $2.53_{\mathrm{c}}^{\mathrm{a}} \pm 0.02$ & $2.75_{\mathrm{d}}^{\mathrm{a}} \pm 0.02$ & $3.36_{\mathrm{e}}^{\mathrm{a}} \pm 0.02$ \\
\hline 3) $80 \mathrm{mg}$ vitamin $\mathrm{E} / \mathrm{kg}$ feed & $0.67_{\mathrm{a}}^{\mathrm{b}} \pm 0.09$ & $0.98_{\mathrm{b}}^{\mathrm{ab}} \pm 0.07$ & $2.27_{\mathrm{c}}^{\mathrm{a}} \pm 0.07$ & $2.52_{\mathrm{d}}^{\mathrm{b}} \pm 0.07$ & $2.82_{\mathrm{e}}^{\mathrm{b}} \pm 0.07$ \\
\hline 4) $120 \mathrm{mg}$ vitamin $\mathrm{E} / \mathrm{kg}$ feed & $0.59^{\mathrm{b}} \pm 0.16$ & $0.79_{b}^{b c} \pm 0.10$ & $1.99_{\mathrm{c}}^{\mathrm{b}} \pm 0.02$ & $2.09_{\mathrm{cd}}^{\mathrm{c}} \pm 0.02$ & $2.13_{\mathrm{d}}^{\mathrm{c}} \pm 0.02$ \\
\hline 5) $160 \mathrm{mg}$ vitamin $\mathrm{E} / \mathrm{kg}$ feed & $0.31_{a}{ }^{c} \pm 0.08$ & $0.59 a^{c} \pm 0.22$ & $0.97_{b}^{c} \pm 0.32$ & $1.02_{b}{ }^{d} \pm 0.22$ & $1.06_{b}{ }^{\mathrm{d}} \pm 0.32$ \\
\hline
\end{tabular}

${ }^{\mathrm{a}}$ Values in the same column within each experiment with different superscripts differ significantly $(\mathrm{P}<0.05)$.

${ }^{\mathrm{a}}$ Values in the same row within each experiment with different subscripts differ significantly $(\mathrm{P}<0.05)$.

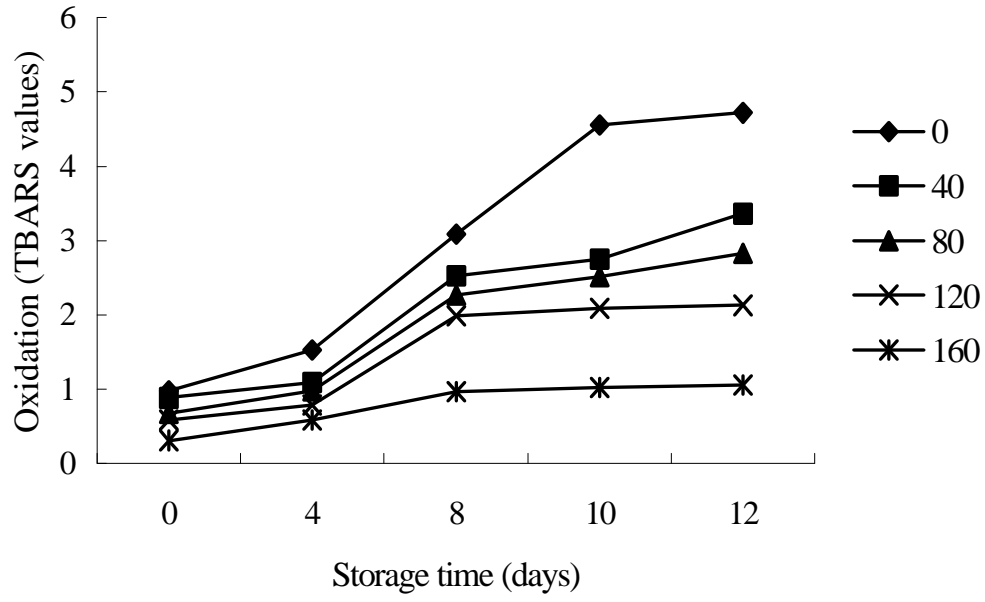

Figure 2 The effect of different levels of vitamin $\mathrm{E}$ ( $\mathrm{mg}$ vitamin $\mathrm{E} / \mathrm{kg}$ feed) on the oxidative stability (TBARS) of broiler carcasses during storage at $4{ }^{\circ} \mathrm{C}$ for up to 12 days

In experiment 1 (frozen storage), the TBARS values of the basal diet were higher $(\mathrm{P}<0.05)$ than any of the other diets supplemented with vitamin E. The two diets containing the highest concentrations of vitamin $\mathrm{E}$ (diet $6-100 \mathrm{mg}$ vitamin $\mathrm{E} / \mathrm{kg}$ feed continuously and diet $11-200 \mathrm{mg}$ vitamin $\mathrm{E} / \mathrm{kg}$ feed, last 3 weeks) had the lowest TBARS values, which did not differ from each other on day 30. After 90 days storage the only diet for which the TBARS value that did not increase $(\mathrm{P}<0.05)$ was that of diet 6 . After 150 days storage the TBARS value of this diet increased $(\mathrm{P}<0.05)$ compared to that at 30 days storage, but did not differ from that at 90 days (Table 4). According to the linear regression equation fitted to the data (Table 5), diet 6 showed the slowest rate of increase $(0.004 / \mathrm{d})$ in TBARS values. In general, TBARS values decreased 
with an increase in dietary vitamin E level (Table 4) for both experiments when compared at the same storage period.

In experiment 2 both the basal diet and diet $2(40 \mathrm{mg}$ vitamin $\mathrm{E} / \mathrm{kg}$ feed $)$ had higher $(\mathrm{P}<0.05)$ TBARS values than the other three diets. Diet 5 (160 mg vitamin E/kg feed) had the lowest TBARS value on day 0 as well as for the rest of the experimental period. The TBARS value of diet 5 on day 4 did not differ from that obtained on day 0 . On day 8 , diet 5 had a TBARS value $(0.97 \pm 0.32)$ similar to that of the basal diet on day $0(0.98 \pm 0.12)$. Diets 3 and 4 had 4-day TBARS values similar and lower (respectively) than that of the basal diet on day 0 (Table 4). Diet 5 also had the slowest rate of increase (Table 5) of TBARS values. As expected, the refrigerated (experiment 2) broiler carcasses had a much higher rate of increase of TBARS values compared to that of the frozen (experiment 1) carcasses (Table 5).

Table 5 Linear regression equations relating storage time to TBARS values for broilers fed different dietary levels of vitamin $\mathrm{E}$ (mean \pm s.e.)

\begin{tabular}{|c|c|c|}
\hline $\begin{array}{c}\text { Equation: } \mathrm{y}=\mathrm{a}+\mathrm{bx} \\
\text { where } \mathrm{x}=\text { storage time (days) } \\
\mathrm{y}=\text { TBARS values }\end{array}$ & $\begin{array}{l}\mathrm{a}=\text { intercept } \\
\mathrm{b}=\text { slope }\end{array}$ & \\
\hline Experiment $1\left(-20^{\circ} \mathrm{C}\right)$ & $\mathrm{a} \pm$ s.e. & $\mathrm{b} \pm$ s.e. \\
\hline 1) Basal & $0.885 \pm 0.331$ & $0.028 \pm 0.003$ \\
\hline 2) $20 \mathrm{mg}$ vitamin $E / \mathrm{kg}$ feed & $0.609 \pm 0.095$ & $0.018 \pm 0.001$ \\
\hline 3) $40 \mathrm{mg}$ vitamin $\mathrm{E} / \mathrm{kg}$ feed & $0.663 \pm 0.113$ & $0.015 \pm 0.001$ \\
\hline 4) $60 \mathrm{mg}$ vitamin $E / \mathrm{kg}$ feed & $0.448 \pm 0.101$ & $0.016 \pm 0.001$ \\
\hline 5) $80 \mathrm{mg}$ vitamin $\mathrm{E} / \mathrm{kg}$ feed & $0.598 \pm 0.146$ & $0.012 \pm 0.001$ \\
\hline 6) $100 \mathrm{mg}$ vitamin $\mathrm{E} / \mathrm{kg}$ feed & $0.566 \pm 0.165$ & $0.004 \pm 0.002$ \\
\hline 7) $120 \mathrm{mg}$ vitamin $\mathrm{E} / \mathrm{kg}$ feed & $0.558 \pm 0.077$ & $0.021 \pm 0.001$ \\
\hline 8) $140 \mathrm{mg}$ vitamin $\mathrm{E} / \mathrm{kg}$ feed & $0.724 \pm 0.138$ & $0.016 \pm 0.001$ \\
\hline 9) $160 \mathrm{mg}$ vitamin $\mathrm{E} / \mathrm{kg}$ feed & $0.488 \pm 0.139$ & $0.016 \pm 0.001$ \\
\hline 10) $180 \mathrm{mg}$ vitamin $\mathrm{E} / \mathrm{kg}$ feed & $0.616 \pm 0.170$ & $0.013 \pm 0.002$ \\
\hline 11) $200 \mathrm{mg}$ vitamin $E / \mathrm{kg}$ feed & $0.552 \pm 0.184$ & $0.009 \pm 0.002$ \\
\hline \multicolumn{3}{|l|}{ Experiment $2\left(4^{\circ} \mathrm{C}\right)$} \\
\hline 1) Basal & $0.639 \pm 0.182$ & $0.344 \pm 0.023$ \\
\hline 2) $40 \mathrm{mg}$ vitamin $E / \mathrm{kg}$ feed & $0.648 \pm 0.103$ & $0.217 \pm 0.013$ \\
\hline 3) $80 \mathrm{mg}$ vitamin $\mathrm{E} / \mathrm{kg}$ feed & $0.522 \pm 0.085$ & $0.196 \pm 0.011$ \\
\hline 4) $120 \mathrm{mg}$ vitamin $\mathrm{E} / \mathrm{kg}$ feed & $0.497 \pm 0.099$ & $0.150 \pm 0.012$ \\
\hline 5) $160 \mathrm{mg}$ vitamin $\mathrm{E} / \mathrm{kg}$ feed & $0.337 \pm 0.097$ & $0.067 \pm 0.012$ \\
\hline
\end{tabular}

The CIELAB (Commission International de l' Eclairage, 1976) colour measurements for experiment 2 are given in Table 6 . There were no differences $(\mathrm{P}>0.05)$ in colour measurements for colours $\mathrm{L}^{*}, \mathrm{a}^{*}$ or $\mathrm{b}^{*}$ between dietary treatments. Different regression equations were fitted for each colour over time and all three colours showed significant $(\mathrm{P}<0.05)$ cubic regressions over time. However, only $10 \%$ of the variation in colour was explained by this regression and it was concluded that the cubic tendency was not biologically significant.

The aerobic plate counts (APC) of experiment 2 are listed in Table 7. The number of cells increased (P $<0.05$ ) over time, regardless of the level of vitamin E supplementation (Figure 3; Table 8). Although some of the APC differed significantly with vitamin E inclusion levels at specific storage periods, no trends were observed that could be attributed to dietary factors. 
Table 6 The effect of various dietary vitamin E levels on the colour deterioration of broiler carcasses kept under refrigerated $\left(4^{\circ} \mathrm{C}\right)$ storage (mean \pm s.e.)

Vitamin E concentration
Experiment $2\left(4^{\circ} \mathrm{C}\right)$
1) Basal
2) $40 \mathrm{mg}$ vitamin $\mathrm{E} / \mathrm{kg}$ feed
3) $80 \mathrm{mg}$ vitamin $\mathrm{E} / \mathrm{kg}$ feed
4) $120 \mathrm{mg}$ vitamin $\mathrm{E} / \mathrm{kg}$ feed
5) $160 \mathrm{mg}$ vitamin $\mathrm{E} / \mathrm{kg}$ feed

1) Basal

2) $40 \mathrm{mg}$ vitamin $\mathrm{E} / \mathrm{kg}$ feed

3) $80 \mathrm{mg}$ vitamin $\mathrm{E} / \mathrm{kg}$ feed

4) $120 \mathrm{mg}$ vitamin $\mathrm{E} / \mathrm{kg}$ feed

5) $160 \mathrm{mg}$ vitamin $\mathrm{E} / \mathrm{kg}$ feed

\begin{tabular}{|c|c|}
\hline Day 0 & Day 4 \\
\hline $22_{\mathrm{a}}^{\mathrm{a}} \pm 1.40$ & $46.14_{\mathrm{a}}^{\mathrm{a}} \pm 2.42$ \\
\hline $70_{\mathrm{ab}}{ }^{\mathrm{a}} \pm 2.43$ & $44.74_{\mathrm{ab}}{ }^{\mathrm{a}} \pm 1.05$ \\
\hline $.72_{\mathrm{a}}^{\mathrm{a}} \pm 1.45$ & $45.21_{\mathrm{a}}^{\mathrm{a}} \pm 3.16$ \\
\hline $02_{\mathrm{a}}{ }^{\mathrm{a}} \pm 3.02$ & $45.34^{a}{ }^{a} \pm 1.39$ \\
\hline $61_{\mathrm{ab}}{ }^{\mathrm{a}} \pm 2.66$ & $42.95_{\mathrm{a}}{ }^{\mathrm{a}} \pm 1.48$ \\
\hline Day 0 & Day 4 \\
\hline $07_{\mathrm{a}}^{\mathrm{a}} \pm 2.38$ & $3.90_{\mathrm{a}}^{\mathrm{a}} \pm 1.05$ \\
\hline $.20_{\mathrm{a}}^{\mathrm{a}} \pm 1.38$ & $4.10_{\mathrm{a}}{ }^{\mathrm{a}} \pm 0.86$ \\
\hline $54_{\mathrm{ab}}{ }^{\mathrm{a}} \pm 2.23$ & $3.86_{a}^{a} \pm 2.16$ \\
\hline $.12_{\mathrm{ab}}{ }^{\mathrm{a}} \pm 0.31$ & $3.76_{\mathrm{a}}^{\mathrm{a}} \pm 1.70$ \\
\hline $.79_{\mathrm{a}}^{\mathrm{a}} \pm 2.42$ & $4.47_{\mathrm{a}}^{\mathrm{a}} \pm 0.80$ \\
\hline
\end{tabular}

$$
\text { Day } 0
$$

$13.91_{\mathrm{a}}^{\mathrm{a}} \pm 0.54$

$13.23_{\mathrm{a}}^{\mathrm{a}} \pm 1.31$

$12.94_{\mathrm{a}}^{\mathrm{a}} \pm 1.42$

$13.93_{\mathrm{a}}^{\mathrm{a}} \pm 0.95$

$14.33_{\mathrm{a}}{ }^{\mathrm{a}} \pm 1.62$

Day 4

$11.96^{a} \pm 1.13$

$11.61_{\mathrm{a}}^{\mathrm{a}} \pm 1.89$

L*

Day 8

$42.48_{\mathrm{b}}{ }^{\mathrm{a}} \pm 1.33$

$42.67 \mathrm{a}^{\mathrm{a}} \pm 2.74$

$43.98_{\mathrm{a}}^{\mathrm{a}} \pm 3.96$

$44.22_{\mathrm{a}}^{\mathrm{a}} \pm 3.88$

$44.90_{\mathrm{ab}}{ }^{\mathrm{a}} \pm 2.06$

$\mathrm{a}^{*}$

\section{Day 8}

$5.97 \mathrm{a}^{\mathrm{a}} \pm 1.43$

$6.33 \mathrm{a}^{\mathrm{a}} \pm 2.50$

$7.00_{\mathrm{b}}^{\mathrm{a}} \pm 2.15$

$4.75_{\mathrm{ab}}{ }^{\mathrm{a}} \pm 1.69$

$5.42 \mathrm{a}^{\mathrm{a}} \pm 1.05$

b*

$13.24_{\mathrm{ab}}{ }^{\mathrm{a}} \pm 0.99$

Day 8

$11.81_{\mathrm{b}}{ }^{\mathrm{a}} \pm 1.38$

$13.91_{a}{ }^{b} \pm 1.27$

$13.06^{a b} \pm 1.96$

$12.82_{\mathrm{a}}^{\mathrm{ab}} \pm 0.84$

$14.01_{\mathrm{a}}^{\mathrm{b}} \pm 1.08$
Day 10

$43.31_{\mathrm{ab}}{ }^{\mathrm{a}} \pm 2.67$

$45.58_{\mathrm{ab}}{ }^{\mathrm{a}} \pm 1.80$

$45.07_{\mathrm{a}}^{\mathrm{a}} \pm 1.23$

$43.97 \mathrm{a}^{\mathrm{a}} \pm 4.37$

$43.39^{a} \pm 1.46$

Day 10

$5.61_{\mathrm{a}}^{\mathrm{ab}} \pm 0.81$

$6.62 \mathrm{a}^{\mathrm{a}} \pm 0.84$

$4.15_{\mathrm{ab}}^{\mathrm{b}} \pm 0.93$

$5.95_{\mathrm{b}}{ }^{\mathrm{a}} \pm 1.45$

$5.34_{\mathrm{a}}^{\mathrm{ab}} \pm 1.16$

Day 10

$13.08_{\mathrm{ab}}{ }^{\mathrm{a}} \pm 2.18$

$13.31_{\mathrm{a}}{ }^{\mathrm{a}} \pm 1.68$

$12.78_{\mathrm{a}}^{\mathrm{a}} \pm 1.16$

$12.61_{\mathrm{a}}{ }^{\mathrm{a}} \pm 2.25$

$11.67 \mathrm{a}^{\mathrm{a}} \pm 2.06$
Day 12

$43.75_{\mathrm{ab}}{ }^{\mathrm{a}} \pm 3.10$

$46.41_{\mathrm{b}}{ }^{\mathrm{a}} \pm 2.51$

$47.59^{a} \pm 2.23$

$46.30_{\mathrm{a}}^{\mathrm{a}} \pm 2.58$

$47.54_{b}{ }^{a} \pm 2.23$

\section{Day12}

$4.72_{\mathrm{a}}{ }^{\mathrm{a}} \pm 1.22$

$5.13_{\mathrm{a}}{ }^{\mathrm{a}} \pm 2.47$

$5.41_{\mathrm{ab}}{ }^{\mathrm{a}} \pm 1.71$

$4.41_{\mathrm{ab}}{ }^{\mathrm{a}} \pm 0.73$

$4.79 a^{a} \pm 0.46$

Day 12

$11.73_{\mathrm{b}}{ }^{\mathrm{a}} \pm 1.17$

$10.74_{b}{ }^{a} \pm 1.62$

$11.61_{\mathrm{a}}^{\mathrm{a}} \pm 1.91$

$12.86_{a}^{a} \pm 1.63$

$12.32_{\mathrm{a}}^{\mathrm{a}} \pm 2.52$

${ }^{\mathrm{a}}$ Values in the same column with different superscripts differ significantly $(\mathrm{P}<0.05)$

${ }^{\text {aV } V a l u e s ~ i n ~ t h e ~ s a m e ~ r o w ~ w i t h ~ d i f f e r e n t ~ s u b s c r i p t s ~ d i f f e r ~ s i g n i f i c a n t l y ~}(\mathrm{P}<0.05)$

Table 7 The effect of various dietary vitamin E levels on the microbiological spoilage (aerobic plate counts $=\log 10)$ of broiler carcasses kept under refrigerated $\left(4^{\circ} \mathrm{C}\right)$ storage $($ mean \pm s.e. $)$

\begin{tabular}{|c|c|c|c|c|}
\hline \multirow{2}{*}{$\begin{array}{l}\text { Vitamin E concentration } \\
\text { Experiment } 2\left(4^{\circ} \mathrm{C}\right)\end{array}$} & \multicolumn{4}{|c|}{ Aerobic plate counts $(\log 10)$} \\
\hline & Day 0 & Day 4 & Day 8 & Day 10 \\
\hline 1) Basal & $4.49_{\mathrm{a}}^{\mathrm{b}} \pm 0.37$ & $7.16_{b}{ }^{a} \pm 0.13$ & $8.19_{b}^{b} \pm 0.17$ & $8.90_{c}^{\mathrm{bc}} \pm 0.23$ \\
\hline 2) $40 \mathrm{mg}$ vitamin $E / \mathrm{kg}$ feed & $5.599^{a} \pm 0.37$ & $7.12_{\mathrm{b}}^{\mathrm{a}} \pm 0.39$ & $7.89^{\mathrm{a}} \pm 0.29$ & $8.80_{d}{ }^{a} \pm 0.19$ \\
\hline 3) $80 \mathrm{mg}$ vitamin $\mathrm{E} / \mathrm{kg}$ feed & $5.10_{\mathrm{a}}^{\mathrm{b}} \pm 0.28$ & $7.15_{b}{ }^{a} \pm 0.32$ & $7.96_{c}^{\mathrm{ab}} \pm 0.28$ & $8.91_{\mathrm{d}}^{\mathrm{ac}} \pm 0.21$ \\
\hline 4) $120 \mathrm{mg}$ vitamin $\mathrm{E} / \mathrm{kg}$ feed & $5.05^{a} a^{a b} \pm 0.23$ & $7.37_{\mathrm{b}}^{\mathrm{a}} \pm 0.06$ & $8.15^{\mathrm{ab}} \pm 0.11$ & $8.40_{d}{ }^{a} \pm 0.16$ \\
\hline 5) $160 \mathrm{mg}$ vitamin $\mathrm{E} / \mathrm{kg}$ feed & $5.33^{a} a^{a b} \pm 0.77$ & $7.37_{\mathrm{b}}^{\mathrm{a}} \pm 0.15$ & $7.60_{c}^{\mathrm{ab}} \pm 0.73$ & $8.58_{d}^{b} \pm 0.11$ \\
\hline
\end{tabular}

${ }^{a}$ Values in the same column with different superscripts differ significantly $(\mathrm{P}<0.05)$.

${ }^{a}$ Values in the same row with different subscripts differ significantly $(\mathrm{P}<0.05)$. 


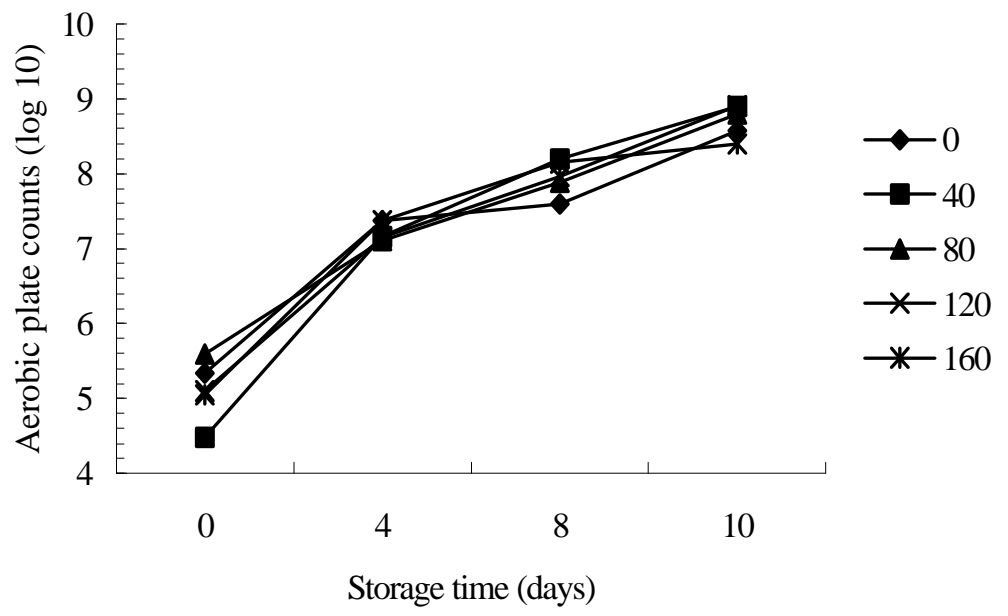

Figure 3 The effect of different levels of vitamin $\mathrm{E}$ (mg vitamin $\mathrm{E} / \mathrm{kg}$ feed) on the microbiological spoilage (aerobic plate counts) of broiler carcasses during storage at $4{ }^{\circ} \mathrm{C}$ for up to 10 days.

Table 8 Linear equations relating refrigerated $\left(4^{\circ} \mathrm{C}\right)$ storage time to APC for broilers fed different dietary levels of vitamin $\mathrm{E}$ (mean \pm s.e.)

\begin{tabular}{lcc}
\hline $\begin{array}{l}\text { Equation: } y=a+b x \\
\text { where } \mathrm{x}=\text { storage time (days) } \\
y=\text { aerobic plate counts (log 10) }\end{array}$ & $\begin{array}{l}\mathrm{a}=\text { intercept } \\
\mathrm{b}=\text { slope }\end{array}$ \\
& $\mathrm{a} \pm$ s.e. & $\mathrm{b} \pm$ s.e. \\
\hline 1) Basal & $5.612 \pm 0.279$ & $0.292 \pm 0.042$ \\
2) $40 \mathrm{mg}$ vitamin E/kg feed & $4.844 \pm 0.199$ & $0.426 \pm 0.030$ \\
3) $80 \mathrm{mg}$ vitamin E/kg feed & $5.677 \pm 0.151$ & $0.304 \pm 0.023$ \\
4) $120 \mathrm{mg}$ vitamin E/kg feed & $5.301 \pm 0.163$ & $0.360 \pm 0.024$ \\
5) $160 \mathrm{mg}$ vitamin E/kg feed & $5.441 \pm 0.197$ & $0.327 \pm 0.029$ \\
\hline
\end{tabular}

The samples from day 0 for experiment 2 were also spiked with the following bacteria: Staphylococcus aureus, Salmonella enteritidis and Listeria innocua. Although the effect of time was not analysed statistically, an increase in bacterial counts was noted over time (Table 9). Bacterial counts did not differ between dietary treatments for the different periods.

Table 9 The effect of various dietary vitamin E levels on the microbiological spoilage (aerobic plate counts $=\log 10)$ of broiler meat samples kept under refrigerated $\left(4{ }^{\circ} \mathrm{C}\right)$ storage and spiked with Staphylococcus, Salmonella and Listeria spp. (mean \pm s.e.)

\begin{tabular}{lccc}
\hline $\begin{array}{l}\text { Vitamin E concentration } \\
\text { Experiment 2 }\left(4^{\circ} \mathrm{C}\right)\end{array}$ & Day 1 & Aerobic plate counts $(\log 10)$ & Day 3 \\
\hline Staphylococcus & & & Day 6 \\
1) Basal & $5.44 \pm 0.143$ & $5.75 \pm 0.213$ & $5.87 \pm 0.810$ \\
2) $80 \mathrm{mg}$ vitamin E/kg feed & $5.54 \pm 0.063$ & $5.86 \pm 0.362$ & $5.48 \pm 0.675$ \\
3) $160 \mathrm{mg}$ vitamin E/kg feed & $5.73 \pm 0.151$ & $5.50 \pm 0.281$ & $6.21 \pm 0.435$ \\
Salmonella & & & \\
1) Basal & $7.55 \pm 0.103$ & $8.65 \pm 0.337$ & $10.24 \pm 0.053$ \\
2) $80 \mathrm{mg}$ vitamin E/kg feed & $7.60 \pm 0.175$ & $8.74 \pm 0.090$ & $9.56 \pm 0.788$ \\
3) $160 \mathrm{mg}$ vitamin E/kg feed & $7.48 \pm 0.000$ & $8.90 \pm 0.137$ & $9.85 \pm 0.026$ \\
Listeria & & & \\
1) Basal & $7.48 \pm 0.000$ & $9.42 \pm 0.344$ & $10.38 \pm 0.077$ \\
2) $80 \mathrm{mg}$ vitamin E/kg feed & $7.58 \pm 0.081$ & $9.52 \pm 0.083$ & $10.54 \pm 0.088$ \\
3) $160 \mathrm{mg}$ vitamin E/kg feed & $7.50 \pm 0.117$ & $9.09 \pm 0.383$ & $10.32 \pm 0.130$ \\
\hline
\end{tabular}

The South African Journal of Animal Science is available online at http://www.sasas.co.za/Sajas.html 
Fatty acid analysis was done on the muscle samples from broilers in experiment 2 for days 0 and 12 . The aim was to monitor changes in the fatty acid profile (a potential decrease in unsaturated fatty acids and increase in saturated fatty acids) over time. There were no differences $(\mathrm{P}>0.05)$ between treatments for the fatty acid categories measured. Both saturated fatty acids (SFA) and polyunsaturated fatty acids (PUFA) in samples from treatment four on day 0 differed $(\mathrm{P}<0.05)$ compared to that of treatments 3 and 5 . None of the individual fatty acids changed $(\mathrm{P}>0.05)$ over time (Appendix 1$)$. Table 10 gives the effect of refrigerated (4 ${ }^{\circ} \mathrm{C}$ ) storage on the fatty acid profile of the main fatty acid groups: saturated fatty acids (SFA), monounsaturated fatty acids (MUFA) and polyunsaturated fatty acids (PUFA).

Table 10 The effect of various dietary levels of vitamin $\mathrm{E}$ on the fatty acid profile of broiler carcasses kept under refrigerated $\left(4{ }^{\circ} \mathrm{C}\right)$ storage (mean \pm s.e. $)$

\begin{tabular}{|c|c|c|c|c|c|c|}
\hline \multirow[t]{3}{*}{ Vitamin E concentration } & \multicolumn{6}{|c|}{ Fatty acid group* } \\
\hline & \multicolumn{2}{|c|}{ SFA } & \multicolumn{2}{|c|}{ MUFA } & \multicolumn{2}{|c|}{ PUFA } \\
\hline & Day 0 & Day 12 & Day 0 & Day 12 & Day 0 & Day 12 \\
\hline \multicolumn{7}{|l|}{ Experiment $2\left(4^{\circ} \mathrm{C}\right)$} \\
\hline 1) Basal & $26_{\mathrm{a}}^{\mathrm{ab}} \pm 0.93$ & $26_{\mathrm{a}}{ }^{\mathrm{a}} \pm 0.54$ & $42_{\mathrm{b}}{ }^{\mathrm{a}} \pm 1.49$ & $41_{\mathrm{b}}{ }^{\mathrm{a}} \pm 2.08$ & $32^{\mathrm{ac}} \pm 1.13$ & $34_{c}{ }^{a} \pm 2.43$ \\
\hline 2) $40 \mathrm{mg}$ vitamin $E / \mathrm{kg}$ feed & $26_{\mathrm{a}}^{\mathrm{ab}} \pm 1.21$ & $25_{\mathrm{a}}^{\mathrm{a}} \pm 1.68$ & $42_{b}{ }^{a} \pm 2.08$ & $41_{\mathrm{b}}{ }^{\mathrm{a}} \pm 3.46$ & $32_{\mathrm{c}}^{\mathrm{ab}} \pm 1.80$ & $33_{\mathrm{c}}{ }^{\mathrm{a}} \pm 3.67$ \\
\hline 3) $80 \mathrm{mg}$ vitamin $\mathrm{E} / \mathrm{kg}$ feed & $26^{\mathrm{b}} \pm 0.77$ & $26_{a}^{a} \pm 1.23$ & $41_{b}{ }^{a} \pm 0.96$ & $39_{b}^{a} \pm 2.02$ & $34_{c}^{b c} \pm 1.36$ & $35_{c}{ }^{a} \pm 1.62$ \\
\hline 4) $120 \mathrm{mg}$ vitamin $\mathrm{E} / \mathrm{kg}$ feed & $27_{\mathrm{a}}^{\mathrm{a}} \pm 1.14$ & $26_{a}^{a} \pm 1.50$ & $42_{\mathrm{b}}{ }^{\mathrm{a}} \pm 1.47$ & $41_{\mathrm{b}}^{\mathrm{a}} \pm 2.12$ & $30_{c}{ }^{\mathrm{a}} \pm 1.81$ & $33_{c}{ }^{a} \pm 3.28$ \\
\hline 5) $160 \mathrm{mg}$ vitamin $\mathrm{E} / \mathrm{kg}$ feed & $25_{\mathrm{a}}^{\mathrm{b}} \pm 0.91$ & $27_{\mathrm{a}}^{\mathrm{a}} \pm 0.75$ & $40_{\mathrm{b}}{ }^{\mathrm{a}} \pm 0.50$ & $39_{\mathrm{b}}{ }^{\mathrm{a}} \pm 1.71$ & $34_{c}^{\mathrm{b}} \pm 1.32$ & $34_{c}{ }^{a} \pm 2.28$ \\
\hline
\end{tabular}

*Fatty acid concentration as a percentage of total fatty acids measured.

${ }^{a}$ Values in the same column with different superscripts differ significantly $(\mathrm{P}<0.05)$.

${ }^{a}$ Values in the same row with different subscripts differ significantly $(\mathrm{P}<0.05)$.

In experiment 2, $\mathrm{pH}$ measurements were taken at $45 \mathrm{~min}, 2,4$ and $24 \mathrm{hrs}$ post-mortem. There were no differences $(\mathrm{P}>0.05)$ in $\mathrm{pH}$ between dietary treatments (Table 11). Different regression equations were fitted and the data showed statistically significant $(\mathrm{P}<0.05)$ cubic regressions over time. However, only $44 \%$ of the variation was explained by this regression. In general, all dietary treatments had a higher final $\mathrm{pH} 24$ than that at 45 min post-mortem.

Table 11 The effect of various dietary vitamin $\mathrm{E}$ levels on the $\mathrm{pH}$ values of broiler carcasses kept under refrigerated $\left(4^{\circ} \mathrm{C}\right)$ storage. (mean \pm s.e.)

\begin{tabular}{|c|c|c|c|c|}
\hline \multirow{2}{*}{ Vitamin E concentration } & \multicolumn{4}{|c|}{$\mathrm{pH}$ value } \\
\hline & $45 \mathrm{~min}$ & $2 \mathrm{hrs}$ & $4 \mathrm{hrs}$ & $24 \mathrm{hrs}$ \\
\hline \multicolumn{5}{|l|}{ Experiment $2\left(4^{\circ} \mathrm{C}\right)$} \\
\hline 1) Basal & $5.76_{c}{ }^{a} \pm 0.225$ & $6.07 \mathrm{ab}{ }^{\mathrm{a}} \pm 0.311$ & $6.03_{b}{ }^{a} \pm 0.234$ & $6.22 a^{a} \pm 0.236$ \\
\hline 2) $40 \mathrm{mg}$ vitamin $E / \mathrm{kg}$ feed & $5.71_{c}^{a} \pm 0.173$ & $6.01_{\mathrm{a}}^{\mathrm{a}} \pm 0.133$ & $6.01_{\mathrm{a}}^{\mathrm{a}} \pm 0.176$ & $6.23_{b}{ }^{a} \pm 0.209$ \\
\hline 3) $80 \mathrm{mg}$ vitamin $E / \mathrm{kg}$ feed & $5.74_{c}{ }^{a} \pm 0.178$ & $6.20^{\mathrm{b}} \pm 0.144$ & $5.98_{\mathrm{b}}{ }^{\mathrm{a}} \pm 0.225$ & $6.29 \mathrm{a}^{\mathrm{a}} \pm 0.175$ \\
\hline 4) $120 \mathrm{mg}$ vitamin $E / \mathrm{kg}$ feed & $5.74_{d}{ }^{a} \pm 0.211$ & $6.09^{a b} \pm 0.174$ & $5.95_{\mathrm{b}}^{\mathrm{a}} \pm 0.119$ & $6.21_{\mathrm{c}}{ }^{\mathrm{a}} \pm 0.199$ \\
\hline 5) $160 \mathrm{mg}$ vitamin E/kg feed & $5.68_{c}{ }^{a} \pm 0.234$ & $6.03^{a}{ }^{a} \pm 0.215$ & $5.97_{\mathrm{a}}^{\mathrm{a}} \pm 0.286$ & $6.23_{\mathrm{b}}{ }^{\mathrm{a}} \pm 0.173$ \\
\hline
\end{tabular}

${ }^{\mathrm{a}}$ Values in the same column with different superscripts differ significantly $(\mathrm{P}<0.05)$.

${ }^{\mathrm{a}}$ Values in the same row with different subscripts differ significantly $(\mathrm{P}<0.05)$.

\section{Discussion}

Table 3 suggests that there was no difference $(\mathrm{P}>0.05)$ in weight gain or feed conversion ratio between levels of dietary vitamin $\mathrm{E}$ supplementation. This observation differs from those of other authors. Kennedy et al. (1991) reported the results of an extensive study on the effects of increased vitamin $\mathrm{E}$ supplementation on profitable commercial broiler production. A total of 168 broiler flocks containing over 3 million birds were studied. The flocks were fed either normal diets or normal diets supplemented with vitamin $\mathrm{E}$ to contain a total concentration of $180 \mathrm{IU} / \mathrm{kg}$ feed throughout their lives. In the broiler flocks receiving the supplemented diets, the feed conversion ratio was improved by $0.8 \%$ and the average weight per bird was increased by $1.4 \%$ compared with those receiving the normal diets. Even after the cost of the 
additional vitamin $\mathrm{E}$ was taken into consideration, the net income per 100 birds was still $2.82 \%$ higher. An explanation for the weight gain and feed conversion results in Table 3 may be that there was no increase in protein or energy utilisation between dietary vitamin E treatments. All dietary treatments were balanced in terms of energy-to-protein ratio. Mcllroy et al. (1993) suggested that high dietary vitamin E concentrations may be most beneficial where there is a challenge to the defence system of the host, and that significantly improved performance would occur more predictably under such conditions. This investigation was performed in an experimental facility where strict hygienic principles are applied (total mortality was $4.2 \%$ and $4.04 \%$ for experiment 1 and 2 respectively), and it is doubtful that the birds were challenged immunologically.

It is generally believed that lipid oxidation in muscle foods is initiated in the highly unsaturated phospholipid fraction in subcellular membranes (Gray \& Pearson, 1987). The autocatalytic peroxidation process probably begins immediately after slaughter. The biochemical changes that accompany postslaughter metabolism and post-mortem ageing in the conversion of muscle to meat give rise to conditions where the process of lipid oxidation is no longer tightly controlled and the balance of pro-oxidative factors/antioxidative capacity favours oxidation. The conversion of muscle to meat is a direct result of the cessation of blood flow and the triggering or arresting of many metabolic processes. Orderly metabolic activity continues during the early post-slaughter period, but because of the cessation of blood flow the product of glycogen breakdown is lactic acid. Lactic acid accumulates in the tissue and gradually lowers the $\mathrm{pH}$ from near neutrality to a more or less mild acid (approximately $\mathrm{pH} 5.5$ ) value. In the post-slaughter phase, it is highly unlikely that the armoury of antioxidant defensive systems available to the cell in the live animal still function because of quantitative changes in several metabolites and physical properties. In many instances, the defensive system may be weakened by nutritional deficiency and the peroxidation process may be greatly accelerated. The rate and extent of oxidation of muscle foods is also likely to be influenced by preslaughter events such as stress, and post-slaughter events such as $\mathrm{pH}$, carcass temperature and cold shortening. In addition, any disruption of the integrity of muscle membranes by mechanical deboning, mincing, restructuring or cooking alters cellular compartmentalisation. This facilitates the interaction of prooxidants with unsaturated fatty acids, resulting in the generation of free radicals and propagation of the oxidative reaction (Asghar et al., 1988).

The susceptibility of muscle tissue to lipid oxidation depends on a number of factors, the most important being the level of polyunsaturated fatty acids present in the particular muscle system (Allen \& Foegeding, 1981). The phospholipids present in subcellular membranes (mitochondria and microsomes) are high in polyunsaturated fatty acids (Gray \& Pearson, 1987) and the vulnerability of membranes to peroxidation is increased because of the close proximity of a range of pro-oxidants.

There is now considerable interest in the antioxidant properties of naturally occurring substances such as vitamin E (Loliger, 1991). Vitamin E, which is usually incorporated in the diet as $\alpha$-tocopheryl acetate, constitutes the second line of antioxidant defence in biological systems, and is the major lipid-soluble antioxidant, breaking the chain of lipid peroxidation in cell membranes and preventing the formation of lipid hydroperoxides (Halliwell, 1987).

Figures 1 and 2 show that TBARS values increased with an increase in storage time. Oxidative changes in muscle foods are generally quantified by the measurement of secondary degradation products. It is accepted that TBARS numbers correlate well with sensory scores of oxidised and warmed-over flavour in muscle food. TBARS numbers greater than 1 correlate significantly with oxidised scores obtained by trained panellists for meats stored under frozen conditions (Buckley \& Morrissey, 1992).

Table 12 Approximate scale for interpretation of TBARS values in meat and meat products (Frigg, undated).

\begin{tabular}{cc}
\hline \multicolumn{2}{c}{ Interpretation of TBARS values } \\
TBARS values & Interpretation \\
\hline$\leq 0.2$ & Good quality \\
$0.2-0.5$ & Limited, tolerable \\
$0.5-1.5$ & Somewhat oxidised \\
$1.5-5.0$ & Oxidised \\
$>5$ & Rancid, non-edible \\
\hline
\end{tabular}


If a TBARS value cut-off point of 1.5 (Table 12) is used in the linear equations in Table 5, storage times for each of the dietary treatments can be estimated (Table 13).

Table 13 The effect of different dietary vitamin E levels on the storage time of broiler carcasses.

\begin{tabular}{|c|c|}
\hline Vitamin $\mathrm{E}$ concentrations & Storage time (days) \\
\hline \multicolumn{2}{|l|}{ Experiment $1\left(-20^{\circ} \mathrm{C}\right)$} \\
\hline 1) Basal & 21 \\
\hline 2) $20 \mathrm{mg}$ vitamin $E / \mathrm{kg}$ feed & 49 \\
\hline 3) $40 \mathrm{mg}$ vitamin $E / \mathrm{kg}$ feed & 56 \\
\hline 4) $60 \mathrm{mg}$ vitamin $\mathrm{E} / \mathrm{kg}$ feed & 66 \\
\hline 5) $80 \mathrm{mg}$ vitamin $\mathrm{E} / \mathrm{kg}$ feed & 75 \\
\hline 6) $100 \mathrm{mg}$ vitamin $\mathrm{E} / \mathrm{kg}$ feed & 234 \\
\hline 7) $120 \mathrm{mg}$ vitamin $\mathrm{E} / \mathrm{kg}$ feed & 45 \\
\hline 8) $140 \mathrm{mg}$ vitamin $\mathrm{E} / \mathrm{kg}$ feed & 49 \\
\hline 9) $160 \mathrm{mg}$ vitamin $\mathrm{E} / \mathrm{kg}$ feed & 63 \\
\hline 10) $180 \mathrm{mg}$ vitamin $E / \mathrm{kg}$ feed & 68 \\
\hline 11) $200 \mathrm{mg}$ vitamin $E / \mathrm{kg}$ feed & 105 \\
\hline \multicolumn{2}{|l|}{ Experiment $2\left(4^{\circ} \mathrm{C}\right)$} \\
\hline 1) Basal & 3 \\
\hline 2) $40 \mathrm{mg}$ vitamin $E / \mathrm{kg}$ feed & 4 \\
\hline 3) $80 \mathrm{mg}$ vitamin $\mathrm{E} / \mathrm{kg}$ feed & 5 \\
\hline 4) $120 \mathrm{mg}$ vitamin $E / \mathrm{kg}$ feed & 7 \\
\hline 5) $160 \mathrm{mg}$ vitamin $E / \mathrm{kg}$ feed & 17 \\
\hline
\end{tabular}

Table 4 shows that none of the meat samples in either treatment 1 or 2 were rancid (TBARS value $>5$ ) before the end of the storage period. The samples from diet 1 (experiment 1) was oxidised from the first day of sampling (one month), suggesting that broiler carcasses fed diets without vitamin E supplementation cannot even be stored for a month at $-20{ }^{\circ} \mathrm{C}$ (Table 13). The samples from treatment 1 (experiment 2) became oxidised at day 3 . It is thus preferable to refrigerate broiler carcasses from birds fed diets without vitamin E supplementation for less than three days before use. Only diet 6 in experiment 1 and diet 5 in experiment 2 provided sufficient protection against oxidation for the length of the storage period. The values given for diet 6 in experiment 1 and diet 5 in experiment 2 (Table 13) are extrapolations of the regression equations and should not be interpreted as actual storage times. From these results it is estimated that, the storage time of broiler carcasses can be doubled under frozen $\left(-20^{\circ} \mathrm{C}\right)$ conditions by supplementation with as little as $20 \mathrm{mg}$ vitamin $\mathrm{E} / \mathrm{kg}$ feed. In the case of refrigerated carcasses, the storage time can be extended by one day by supplementing with $40 \mathrm{mg}$ vitamin E/kg feed. However, further studies on the chemical measurements of lipid oxidation and sensory characteristics are required before final recommendations on optimum dietary levels of vitamin E supplementation can be made.

Table 4 suggests that the samples from diets 7 to 10 (fed in last three weeks) in experiment 1 became oxidised at a faster rate than those from diets 2 to 5 (fed throughout the trial), but this difference was not statistically significant. However, diet 6 differed significantly $(\mathrm{P}<0.05)$ from diet 11 . It can be speculated that the inclusion time of vitamin $\mathrm{E}$ in the feed only becomes important when high levels of vitamin $\mathrm{E}$ are fed continuously. Bartov \& Frigg (1992) showed that oxidative stability was best in tissues from broilers that received vitamin $\mathrm{E}$ continuously during the trial and presumably had higher $\alpha$-tocopherol contents in the meat. This further demonstrates the importance of the equilibrium of vitamin $E$ in the tissue, particularly in membrane structures. Measures taken to reduce the propagation of radicals are stochastic processes. This means that antioxidants have a certain chance or probability to trap a radical according to its chemical characteristics, or because of its specific distribution pattern. Therefore, a chemically suitable antioxidant can only act efficiently if it is not too distant from the place of appearance of a radical. Otherwise, the radical could cause severe damage and propagate in a chain-reaction-like manner before being trapped. The antioxidant, for example $\alpha$-tocopherol, has to be present in an adequate amount and in the appropriate tissue structure. It is speculated that diets 2 to 5 , despite being fed/supplemented throughout the trial, contributed the same amount of vitamin $\mathrm{E}$ to the tissues as diets 7 to 10, which were fed/supplemented only at the end of the trial. 
Short-term feeding of broilers with $160 \mathrm{IU} \alpha$-tocopherol $/ \mathrm{kg}$ for the last five days prior to slaughter was effective in retarding the onset of rancidity in raw whole breast muscle (Marusich et al., 1975), suggesting that a high level of vitamin E supplementation to the finishing diet could be an alternative to continuous supplementation. However, some authors have questioned whether short-term supplementation would guarantee adequate stability in processed muscle considering the relatively slow uptake of $\alpha$-tocopherol into chicken muscle compared to other tissues (Sheehy et al., 1991) and the apparent requirement for deposition of the vitamin in specific locations within the muscle membranes for optimum protection. Bartov \& Frigg (1992) fed broilers a basal diet containing no added vitamin E for 7 weeks (treatment 1), the basal diet supplemented with $100 \mathrm{mg}$ vitamin E/kg for 7 weeks (treatment 2), the basal diet supplemented with 150 $\mathrm{mg} / \mathrm{kg}$ for three weeks and basal diet alone until seven weeks (treatment 3), basal diet with $150 \mathrm{mg} / \mathrm{kg}, 0$ and $100 \mathrm{mg} / \mathrm{kg}$ until week 3, from weeks 3-6 and from weeks 6-7 (treatment 4) and the basal diet alone for 5 weeks and then the basal diet plus $100 \mathrm{mg} / \mathrm{kg}$ until seven weeks (treatment 5). The stability of meat from treatments 3, 4 and 5 was greater than that from treatment 1 (no vitamin E) but was lower than that from treatment 2 (vitamin E given continuously). Brandon et al. (1993) gave one group of broilers a basal diet containing $30 \mathrm{mg} \alpha$-tocopheryl acetate $/ \mathrm{kg}$ feed continuously up to slaughter at six weeks, while other groups were given a supplemented diet containing $200 \mathrm{mg} \alpha$-tocopheryl acetate/kg for $1,2,3,4$ or 5 weeks immediately prior to slaughter. The $\alpha$-tocopherol content of leg and breast muscle increased as the preslaughter supplementation period increased from 0 to 5 weeks. Supplementation of broiler diets with $200 \mathrm{mg}$ $\alpha$-tocopheryl acetate $/ \mathrm{kg}$ for five weeks prior to slaughter improved the oxidative stability of ground muscle during refrigerated and frozen storage and protected against the pro-oxidant effect of salt. These results suggest that a pre-slaughter supplementation period of at least 4-5 weeks, feeding $200 \mathrm{mg} \alpha$-tocopheryl acetate/kg (i.e. 20 times higher than the NRC requirement), is necessary to attain the optimum protective benefit of $\alpha$ - tocopherol in processed meat. Short-term supplementation before slaughter can therefore always give a relative improvement, but more can be achieved with steady state conditions.

The rate of discolouration of meat is related to the effectiveness of oxidation processes and enzymatic reducing systems in controlling metmyoglobin levels in meat (Chan et al., 1995). There were no differences in colour measurements for $\mathrm{L}^{*}, \mathrm{a}^{*}$ or $\mathrm{b}^{*}$ values (Table 5). This result corresponds with those of other authors. Santé \& Lacourt (1994) found that lightness ( $\left.\mathrm{L}^{*}\right)$ did not change over seven days of storage for turkey meat. Dirinck et al. (1996) found that although supplemented pork samples (200 ppm vitamin E) were redder than control samples, the differences were low and non-significant. Cannon et al. (1996) reported that vitamin E supplementation reduced lipid peroxidation in fresh pork but did not influence pork colour. Lanari et al. (1995) reported that, for pork, supplementation had a positive effect only during illuminated storage. The results of this investigation may be due to the fact that colour measurements were taken on the breast muscle (a white muscle), which contains low concentrations of myoglobin and therefore colour degradation could not be detected.

Extended storage accelerated bacterial growth in broilers (Figure 3). In experiment 2, vitamin E supplementation did not influence the bacterial load. These results correspond with data from Chan et al. (1995) and Zerby et al. (1999). Table 7 shows that the microbial counts of the samples of day 0 were already above legal limits $\left(>10^{3} \mathrm{cfu} \mathrm{g}^{-1}\right)$. This may be the result of slaughter conditions, as the broilers were not slaughtered in a commercial abattoir with strict health regulations.

There is now considerable emphasis on modification of the fatty acid composition of animal tissues in an attempt to produce new 'designer' or 'functional' foods. However, increasing the degree of unsaturation of the muscle membranes by dietary manipulation reduces oxidative stability (Monahan et al., 1992). Saturated fatty acids, being composed of paraffinic hydrocarbon chains, are generally considered to have very low reactivity. Introduction of one or more double bonds into a fatty acid provides an active centre that can be the site of a variety of undesirable reactions, as in the case of oxidation. Even though saturated fatty acids can be oxidised, the rate of oxidation greatly depends on the degree of unsaturation. The relative stability of oxidation at $100{ }^{\circ} \mathrm{C}$ has been given as follows: saturated fatty acids 0.8 , oleic acid 1.1 , linoleic acid 13.7 and linolenic acid 25.5. In the series of 18-carbon-atom fatty acids - 18:0, 18:1, 18:2, 18:3 - the relative rate of oxidation has been reported to be in the ratio of 1:100:1200:2500 (DeMan, 1992).

There were no differences $(\mathrm{P}>0.05)$ between dietary treatments for any of the fatty acids measured. Therefore only the main fatty acid groups: saturated fatty acids (SFA), monounsaturated fatty acids (MUFA) and polyunsaturated fatty acids (PUFA) are presented in Table 10. As in the case of colour deterioration in samples with a higher PUFA content, broiler phospholipids could be more susceptible to oxidation and it is 
possible that vitamin E may be needed in greater quantities to affect fatty acid stability. None of the fatty acids changed over time. It was expected that the longer chain PUFA would have been broken down first into short chain SFA, resulting in fewer PUFA and more SFA on day 12. This was not detected in the fatty acid analysis of day 12 samples. However, it can be postulated that there was breakdown of PUFA, MUFA and SFA (in that order of occurrence), but that the breakdown was complete on day 12, resulting only in secondary oxidation products. Because the fatty acids were identified as a percentage of total fatty acids measured, no difference could be detected between the three main groups. The results would have been more accurate if the fatty acids were measured as $\mathrm{mg}$ fatty acid/g of tissue. Inclusion of the samples of days 4,8 and 10 may also have given a better indication of fatty acid breakdown.

There were no differences $(\mathrm{P}>0.05)$ in $\mathrm{pH}$ between dietary groups (Table 11). The data showed a significant $(\mathrm{P}<0.05)$ cubic tendency over time. At $45 \mathrm{~min}$ post-slaughter the $\mathrm{pH}$ values were approximately 5.5 (Table 11). This is the result of irreversible anaerobic glycolysis, which occurs when oxygen is permanently removed from the muscle at death (Lawrie, 1991). The resultant lactic acid production causes the $\mathrm{pH}$ to fall to a value of 5.5. From $45 \mathrm{~min}$ to $2 \mathrm{hrs}$ post-mortem there was a slight increase in muscle $\mathrm{pH}$, which was related to deamination reactions, as reported by Gill (1986). Deamination of amino acids, with liberation of ammonia, results in an increase in $\mathrm{pH}$. However, this reaction is reversible and the release of the hydrogen atom from ammonia causes the $\mathrm{pH}$ to fall 2-4 hrs post-mortem (Table 11). At $24 \mathrm{hrs}$ post-mortem lactic acid degradation stops while deamination continues, resulting in an increase of $\mathrm{pH}$ (Table 11).

\section{Conclusions}

It was concluded that vitamin E supplementation of broiler feed increases the oxidative stability of broiler carcasses under frozen and refrigerated storage. Carcasses of broilers from birds fed nonsupplemented diets could not be stored under frozen conditions for up to one month, whereas nonsupplemented refrigerated carcasses could only be stored for three days. Under frozen conditions supplementation of as little as $20 \mathrm{mg}$ vitamin E/ $\mathrm{kg}$ feed doubled the storage time, whereas supplementation of $40 \mathrm{mg}$ vitamin $\mathrm{E} / \mathrm{kg}$ feed extended storage time by one day in refrigerated broiler carcasses. If this concentration is increased to $160 \mathrm{mg}$ vitamin E/kg feed, a TBARS value similar to that of broilers receiving no vitamin $\mathrm{E}$ will only be reached after eight days of storage at $4{ }^{\circ} \mathrm{C}$. This may have important economic implications for the retail industry. This investigation further showed that vitamin $\mathrm{E}$ supplementation under these conditions had no significant effect on broiler performance, microbial spoilage, colour deterioration, fatty acid composition or post-mortem $\mathrm{pH}$ changes.

\section{Acknowledgements}

Vitamin E was supplied by Hoffmann La-Roche, Basel, Switzerland.

\section{References}

Allen, C. E. \& Foegeding, E. A., 1981. Some lipid characteristics and interactions in muscle foods - review. Food Tech. 35 (5), 253-257.

Asghar, A., Gray, J. I., Buckley, D. J., Pearson, A. M. \& Booren, A. M., 1988. Perspectives in warmed-over flavour. Food Tech. 42 (6), 102-108.

Bartov, I. \& Frigg, M., 1992. Effect of high concentrations of dietary vitamin E during various age periods on performance, plasma vitamin $\mathrm{E}$ and meat stability of broiler chicks at 7 weeks of age. Br. Poultry Sci. 33, 393-402.

Brandon, S., Morrissey, P. A., Buckley, D. J. \& Frigg, M., 1993. Influence of dietary $\alpha$-tocopherol acetate on the oxidative stability of chicken tissues. In: Proceedings of the $11^{\text {th }}$ European Symposium on the Quality of Poultry Meat, (Eds. P. Colin, J. Culioli and F. H. Ricard), pp. 397-403.

Buckley, D. J. \& Morrissey, P. A., 1992. Animal production highlights. In: Vitamin E and Meat Quality, Hoffmann-La Roche Ltd., Basel, Switserland, pp. 24-27.

Buettner, G. R., 1993. The pecking order of free radicals and antioxidants: lipid peroxidation, $\alpha$-tocopherol and ascorbate. Arch. of Biochem. and Biophys. 300, 535-543.

Cannon, J. E., Morgan, J. B., Schmidt, G. R., Tatum, J. D., Sofos, J. N., Smith, G. C., Delmore, R. J. \& Williams, S. N., 1996. Growth and fresh meat quality characteristics of pigs supplemented with vitamin E. J. of Anim. Sci. 74, 98-105. 
Chan, W. K. M., Hakkarainen, K., Faustman, C., Schaefer, D. M. Scheller, K. K. \& Liu, Q., 1995. Colour stability and microbial growth relationships in beef as affected by endogenous $\alpha$-tocopherol. J. of Food Sci. 60 (5), 996-971.

Commission International De L' Eclairage, 1976. Colorimetry. $2^{\text {nd }}$ Ed. CIE, Vienna.

DeMan, J. M., 1992. Chemical and physical properties of fatty acids. In: Fatty Acids in Foods and their Health Implications. (Ed. C. K. Chow), Marcel Dekker Inc., New York, pp.17-45.

Dirinck, P., De Winne, A., Casteels, M. \& Frigg, M., 1996. Studies on vitamin E and meat quality. 1. Effect of feeding high vitamin E levels on time-related pork quality. J. of Agric. and Food Chem. 44, 65-68.

Frigg, M., undated. Research experiences with vitamin E for poultry meat quality. Hoffmann-La Roche Ltd., Basel, Switserland, pp.2.

Genstat 5, Release 3.2, 1997. Genstat 5 Committee of the Statistics department, Rothamsted Experimental Station.

Gill, C. O., 1986. The control of microbial spoilage in fresh meats. In: Advances in Meat Research. (Ed. R. A. Lawrie), Westport, CT: AVI, Vol. 2, p. 49-88.

Gray, J. I. \& Pearson, A. M., 1987. Rancidity and warmed-over flavour. Adv. in Meat Res. 3: 221-269.

Halliwell, B., 1987. Oxidants and disease: some new concepts. FASEB J. 1, 358-364.

Igene, J. O. \& Pearson, A. M., 1979. Role of phospholipids and triglycerides in warmed-over flavour development in meat model systems. J. of Food Sci. 44, 1285-1290.

Kennedy, D. G., Rice, D. A., Bruce, E. A., Goodall, E. A. \& McIlroy, S. G., 1992. Economic effects of increased vitamin E supplementation of broiler diets on commercial broiler production. Br. Poultry Sci. 33, 1015-1023.

Kennedy, D. G., Goodall, E. A., McIlroy, S. G., Bruce, D. W. \& Rice, D. A., 1991. The effects of increased vitamin E supplementation on profitable commercial broiler production. Proc. of the Nutr. Soc. 50, 197 A.

Lanari, M. C., Schaefer, D. M. \& Scheller, K. K., 1995. Dietary vitamin E supplementation and discolouration of pork bone and muscle following modified atmosphere packaging. Meat Sci. 41, 237250.

Lawrie, R. A., 1991. Chemical and Biochemical Constitution of Muscle. In: Meat Science. (Ed. R. A. Lawrie), Pergamon Press, pp. 56-58.

Loliger, J., 1991. The use of antioxidants in foods. In: Free Radicals and Food Additives. (Eds. O. I. Arouma and B. Halliwell), Taylor Francis, London.

Machlin, L. J., 1984. Handbook of vitamins: Nutritional, Biochemical and Clinical Aspects. (Ed. L. J. Machlin), Marcel Dekker Inc., New York, N. Y., pp. 99.

Marusich, W. L., DeRitter, E., Ogring, E. F., Keating, J., Mitrovic, M. \& Bunnell, R. H., 1975. Effect of supplemental vitamin E in control of rancidity in poultry meat. Poultry Sci. 54, 831-844.

McIlroy, S. G., Goodall, E. A., Rice, D. A., McNulty, M. S. \& Kennedy, D. G., 1993. Improved performance in commercial flocks with subclinical infectious bursal disease when fed diets containing increased concentrations of vitamin E. Avian Path. 22, 81-94.

Monahan, F. J., Buckley, D. J., Morrissey, P. A., Lynch, P. B. \& Gray, J. I., 1992. Influence of dietary fat and $\alpha$-tocopherol supplementation in lipid oxidation in pork. Meat Sci. 31, 229-241.

Morrison, W. R. \& Smith, M. L., 1964. Preparation of fatty acid methyl esters and dimethylacetals from lipids with boron fluoride-methanol. J. of Lipid Res. 5, 600-602.

Morrissey, P. A., Buckley, D. J., Sheehy, P. J. A. \& Monahan, F. J., 1994. Vitamin E and meat quality. Proc. of the Nutr. Soc. 53, 289-295.

Pearson, A. M., Gray, J. I., Wolzak, A. M. \& Horenstein, N. A., 1983. Safety implications of oxidised lipids in foods. Food Tech. 37, 121-129.

Santé, V. \& Lacourt, A., 1994.The effect of dietary $\alpha$-tocopherol supplementation and antioxidant spraying on colour stability and lipid oxidation of turkey meat. J of Sci. Food and Agric. 665, 503-507.

Sheehy, P. J. A., Morrissey, P. A. \& Flynn, A., 1993. Influence of heated vegetable oils and alpha-tocopherol acetate supplementation on alpha-tocopherol, fatty acids and lipid peroxidation in chicken muscle. Br. Poultry Sci. 34, 367-381.

Sheehy, P. J. A., Morrissey, P. A. \& Flynn, A., 1991. Influence of dietary $\alpha$-tocopherol on tocopherol concentrations in chick tissues. Brit. Poultry Sci. 32, 391-397. 
Sheldon, B. W., Curtis, P. A., Dawson, P. L. \& Ferket, P. R., 1997. Effect of dietary vitamin E on the oxidative stability, flavour, colour and volatile profiles of refrigerated and frozen turkey breast meat. Poultry Sci. 76, 634-641.

Uchiyama, M. \& Mihara, M., 1978. Determination of malonaldehyde precursor in tissues by thiobarbituric acid test. Anal. Biochem. 86, 271-278.

Zerby, H. N., Belk, K. E., Ahola, J. K., Sofos, J. N., Schaefer, D. M., Morgan, J. B. \& Smith, G. C., 1999. Effects of muscle $\alpha$-tocopherol level and surface microbiological contamination on retail caselife of fresh beef from the US, Japan and Australia. Meat Sci. 52, 111-118. 


\section{Appendix 1}

Table 1 The effect of various dietary vitamin E levels on the fatty acid profile of broiler carcasses kept under refrigerated $\left(4^{\circ} \mathrm{C}\right)$ storage (mean \pm s.e.)

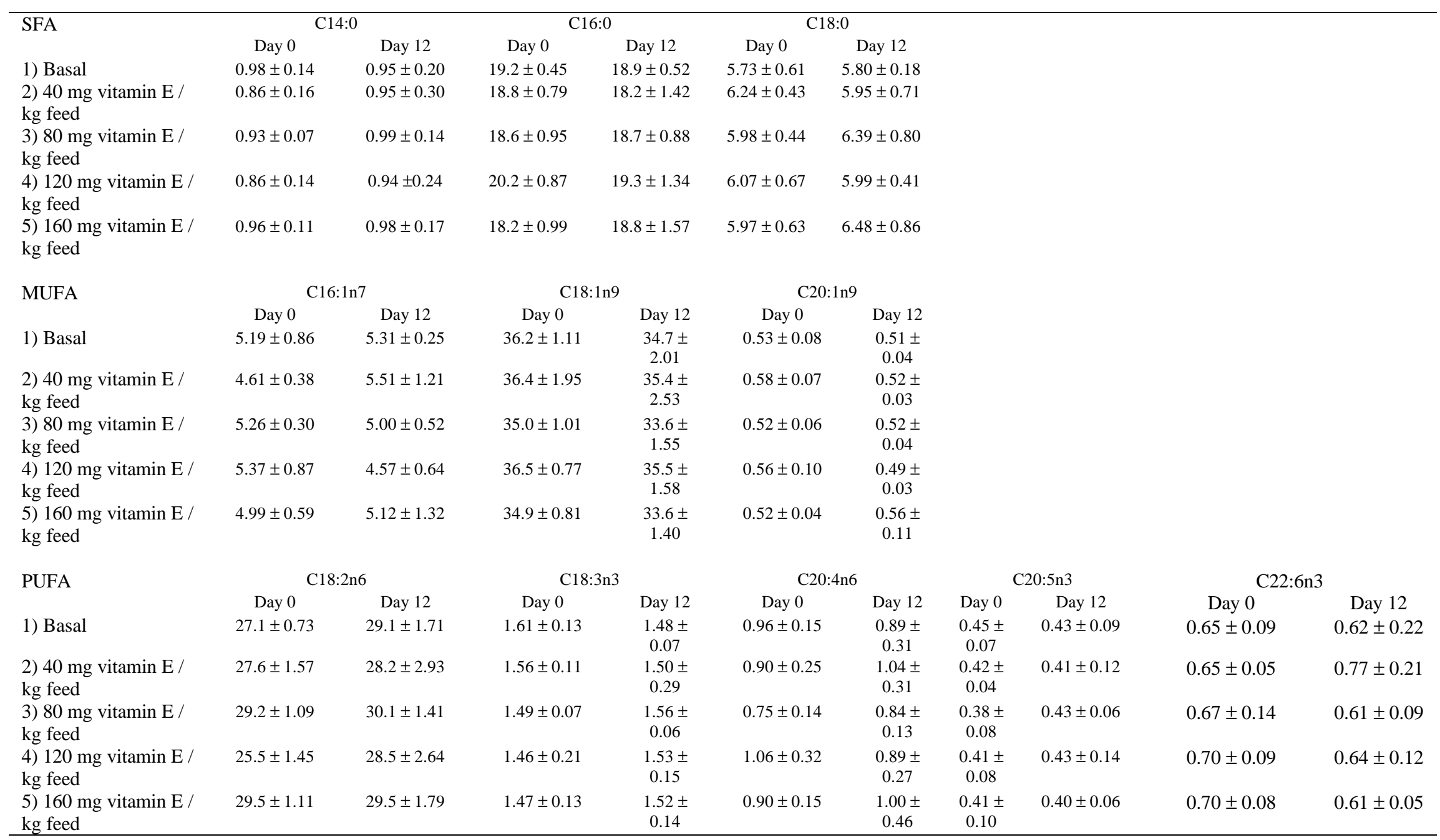

